\title{
A Comparative Study of Pseudomonas pseudomallei and Bacillus mallei
}

\author{
By M. S. REDFEARN, N. J. PALLERONI AND R. Y. STANIER \\ Naval Biological Laboratory, School of Public Health and Department of \\ Bacteriology and Immunology, University of California, Berkeley, California, U.S.A.
}

(Received 2 October 1965)

\begin{abstract}
SUMMARY
A comparative study of many strains of Pseudomonas pseudomallei and Bacillus mallei has shown that these two species are very similar with respect to their nutritional and biochemical properties, thus confirming earlier claims of a relationship between them, based on such criteria as pathological and serological properties. $P$. pseudomallei is in all respects a typical and nutritionally highly versatile member of the genus $P$ seudomonas. In view of this fact we propose that $B$. malle $i$ should also be placed in the genus Pseudomonas, even though it is a permanently non-motile bacterium. The ecology and possible evolutionary relationships between the two species are discussed in the light of the present findings.
\end{abstract}

\section{INTRODUCTION}

The etiological agent of glanders, which is primarily a disease of horses, was discovered in 1882 by Loeffler \& Schütz, and subsequently named Bacillus mallei by Zopf (1885). Like certain other non-motile Gram-negative rod-shaped bacteria which are pathogenic for animals, $B$. mallei has spent much of its scientific career in search of a satisfactory generic location. It has been placed at various times in Pfeifferella (Buchanan, 1918), Malleomyces (Pribram, 1933), Actinobacillus (Brumpt, 1910), Loefflerella (Holden, 1935) and Acinetobacter (Steel \& Cowan, 1964). All these genera are poorly defined pigeon holes for non-motile Gram-negative rod-shaped bacteria of uncertain affinities and mostly of pathogenic propensities.

One other Gram-negative true bacterium has long been recognized to resemble Bacillus mallei. This is $\boldsymbol{B}$. pseudomallei, the etiological agent of melioidosis, a glanders-like disease of man and other mammals which occurs in some regions of the tropics. B. pseudomallei was discovered and described by Whitmore (1913), and his choice of a specific name was determined by its resemblances in pathological and cultural respects to the agent of glanders. Subsequently, Stanton \& Fletcher (1925) and Cravitz \& Miller (1950) showed that there are also serological relationships between these two bacterial species. For several decades the systematic position of $B$. pseudomallei also remained obscure, and it followed $B$. malle $i$ through Malleomyces, Loefferella and Pfeifferella. However, B. pseudomallei is a motile organism, and several workers (Brindle \& Cowan, 1951; Lajudie, Fournier \& Chambon, 1953; Wetmore \& Gochenour, 1956) have shown that it possesses polar multitrichous flagella. Several studies, of which the most thorough is that of Wetmore \& Gochenour (1956), have indicated that B. pseudomallei also resembles 
aerobic pseudomonads (Pseudomonas aeruginosa, $\boldsymbol{P}$. stutzeri) in some cultural and physiological respects. In the 6th edition of Bergey's Manual, Haynes (1957) formally included this organism in the genus Pseudomonas as $P$. pseudomallei. This generic assignation accords with the fragmentary existing information about its nutritional and biochemical properties. Levine, Dowling, Evenson \& Lien (1954) showed that it requires no growth factors, and can grow well in a simple chemically defined medium with several amino acids and organic acids as sources of carbon and energy. Bokman, Levine \& Lusby (1957) found that it metabolizes glucose, probably through the pathway of Entner \& Doudoroff (1952); this is the characteristic mode of glucose dissimilation by several species of aerobic pseudomonads. Finally, Levine \& Wolochow (1960) showed that this organism accumulates poly- $\beta$-hydroxybutyrate as a cellular reserve material, a trait that is also not uncommon in non-fluorescent species among the aerobic pseudomonads (Forsyth, Hayward \& Roberts, 1958).

Bacillus mallei was placed in the genus Actinobacillus by Haupt (1957) in the 7th edition of Bergey's Manual. Since then, an additional significant biological resemblance between Pseudomonas pseudomallei and $\boldsymbol{B}$. mallei has been discovered. Smith \& Cherry (1957) reported that several temperate phages isolated from $P$. pseudomallei could lyse strains of $\boldsymbol{B}$. mallei but not strains of other Pseudomonas species.

As an adjunct to the taxonomic analysis of the aerobic pseudomonads undertaken by Stanier, Palleroni \& Doudoroff (1966), it appeared desirable to characterize Pseudomonas pseudomallei by the same methods; and in view of the indications that Bacillus mallei might be a closely related bacterium, it also was examined.

\section{METHODS}

The methods used have been fully described by Stanier et al. (1966). The routine temperature of incubation was $32^{\circ}$. The results of cultural and physiological tests with Bacillus malle $i$ were recorded only after incubation for 10 days, made necessary by the very slow growth of this organism.

The strains examined were received at the Naval Biological Laboratory of the University of California between 1949 and 1963 and subsequently maintained as lyophil preparations ( Drs R. J. Heckley \& M. M. Johnston, personal communication). Their designations and histories follow.

\section{Pseudomonas pseudomallei}

NBL 101. Received 1949 from Army Medical Service Graduate School, Washington, D.C., U.S.A., as strain 1096.

NBL 102. Received 1951 from same source as 101. Strain 295 of Calcutta School of Tropical Medicine (CSTM); strain MP-F of Wetmore \& Gochenour (1956).

NBL 103. Received 1951 from same source as 101. Strain 294 of CSTM; strain MP-G of Wetmore \& Gochenour (1956).

NBL 104. Received 1951 from the same source as 101. Strain China 3; strain MP-H of Wetmore \& Gochenour (1956).

NBL 111. Received 1953 from Institute of Medical Research (IMR), Kuala Lumpur, as strain Wong Fook Mook. Isolated from human infection in Malaya. 
NBL 112. Same source as 111; IMR strain P.w., isolated from human infection in Malaya.

NBL 113. Same source as 111; IMR strain Horse, isolated 1947-49 from equine infection in Malaya. Possibly identical with strain MP-E of Wetmore \& Gochenour (1956) and that isolated by Davie \& Wells (1952).

NBL 114. Same source as 111. IMR strain Ipoh, isolated from human infection in Malaya.

NBL 115. Received 1953 from Microbiology Division, Fort Detrick, Md., U.S.A., as strain AHS-I. Identical with NCTC 8016; referred to by Brindle \& Cowan (1951). Isolated 1949 from infected sheep in Australia and probably from the outbreak described by Cottew (1950)

NBL 116. Received 1954 from Microbiology Division, Fort Detrick, Md., U.S.A., as AMGS 1454. All strains with AMGS prefix originated from Army Medical Service Graduate School, Washington, D.C., U.S.A., and are probably human isolates. Isolated 1953 in Thailand.

NBL 117. Same source as 116. Strain AMGS 1455, isolated 1954 in Thailand.

NBL 118. Same source as 116. Strain AMGs 1456, isolated 1954 in Thailand.

NBL 119. Same source as 116. Strain AMGS 1457, isolated 1953 in Thailand.

NBL 120. Same source as 116. Strain AMGs 1458, isolated 1953 in Thailand.

NBL 121. Received 1956 as strain 286 from Division of Veterinary Medicine, Walter Reed Army Institute of Research, Washington, D.C., U.S.A. Isolated 1953 from human infection in U.S.A. (Ziskind, Pizzolato, \& Buff, 1954). Strain MP-s of Wetmore \& Gochenour (1956).

NBL 122. Received as strain 56-B-I from same source as 121. Isolated 1946 from human infection in U.S.A. (Gutner \& Fisher, 1948). Strain MP-R of Wetmore \& Gochenour (1956).

NBL 124. Received 1957 from Institüt voor Bacteriologie, Rijksuniversiteit, Utrecht, Netherlands, as Aruba Strain II. Isolated 1955 from infected sheep in Netherlands Antilles. (Sutmöller, Kraneveld \& Schaff, 1957).

NBL 125. Same source as 124 . Isolated 1954 from human infection in Indonesia.

NBL 126. Same source as 124. Aruba strain 2, with same isolation history as 124.

NBL 128. Received 1958 from Division of Veterinary Medicine, Walter Reed Army Institute of Research, as strain $M$ 1379. Isolated 1957 from human infection U.S.A.

NBL 129. Same origin as 128. Strain M 1444, isolated 1957 from human infection in Panama.

NBL 130. Received from Microbiology Division, Fort Detrick, as strain P.w. 124/61. Isolated 1961 from infected pig in Malaya.

NBL 133. Same source as 130. Strain P.w. 140/60, isolated 1960 from infected horse in Malaya.

NBL 135. Same source as 130 . Strain P.w. 62/61, isolated 1961 from infected goat in Malaya.

NBL 137. Received 1963 from Communicable Diseases Center, Atlanta, Ga., U.S.A., as strain 720. Isolated 1962 from human infection in Ecuador (Biegeleisen, Mosquera \& Cherry, 1964).

NBL 138. Same origin as 137. Strain 1960. 
Of the strains originally isolated in the Americas, three (NBL 121, 122, 128), which were isolated from human infections in the U.S.A., almost certainly represent instances of primary infections contracted in the Far East. Strains isolated in the Caribbean region (NBL 124, 126, 129) and in Equador (NBL 137, 138) originated from primary infections contracted in those geographical areas.

\section{Bacillus mallei}

NBL 1. Received 1951 from Professor G. B. Reed, Queens University, Canada, as strain $280 \mathrm{~A}$.

NBL 2. Received 1951 from Army Medical Services Graduate School, Washington, D.C., U.S.A., as strain China (Kweiyang) 3. Originally from the China National Epidemic Prevention Bureau. Isolated 1942 from infected horse.

NBL. 4 Same source as NBL 2. Strain China (Kweiyang) 5, isolated 1942 from infected horse 29.

NBL 5. Same source as NBL 2. Strain 2023. Probably isolated from horse.

NBL 6. Same source and isolation as NBL 5. Strain 2024.

NBL 7. Same source as NBL 2. Strain 3873; strain China 7. Isolated 1944 from human infection.

NBL 8. Received 1951 from Division of Microbiology, Fort Detrick, Md., U.S.A., as strain $\mathrm{K}$ (China 6).

NBL 10. Same source as NBL 1. Strain 3873-18, identical in origin with NBL 7 .

NBL 11. Same source as NBL 1. Strain 3873-18-3-AP, identical in origin with NBL 10. but subjected to three passages through the hamster.

NBL 12. Received 1952 from Division of Microbiology, Fort Detrick, Md., U.S.A., as strain $120 \mathrm{~A}$.

NBL 16. Received 1952 from Captain Turgut Tulgo, Army Veterinary Laboratory, Ankara, Turkey, as strain v. Isolated 1949 from human infection in Turkey.

NBL 17. Same source as NBL 16. Strain vir. Isolated 1951 from human infection in Turkey.

NBL 18. Same source as NBL 16. Strain virr. Isolated 1951 from human infection in Turkey.

NBL 19. Same source as NBL 16. Strain Ix. Isolated 1951 from human infection in Turkey.

NBL 20. Received from National Collection of Type Cultures, England (strain 3709) under the designation Loefflerella mallei (Acinetobacter mallei).

\section{RESULTS}

\section{The characterization of Pseudomonas pseudomallei}

The form and colour of the growth on solid media are high variable in Pseudomonas pseudomallei. Colonies can range in structure from extreme rough to mucoid, and in colour from cream to bright orange (Nigg, Ruch, Scott \& Noble, 1956). Many different combinations of these two characters were represented among the 26 strains examined. In other respects, however, the strains were remarkably uniform; and the few minor differences in nutritional characters that were observed showed no correlations with differences in pigmentation or colony form.

Except as specifically noted, the following characters are shared by all strains. 
Poly- $\beta$-hydroxybutyric acid is accumulated as a cellular reserve material. There is no growth under strictly anaerobic conditions in a complex medium containing glucose. There is abundant growth under strictly anaerobic conditions in a complex medium containing nitrate; and with the exception of strains NBL 104 and 125, growth is accompanied by gas production. Growth occurs at $42^{\circ}$, but not at $5^{\circ}$. Growth is excellent in chemically defined media containing ammonia as sole nitrogen source. Gelatin, starch, Tween 80 and poly- $\beta$-hydroxybutyric acid are hydrolysed by extracellular enzymes.

Five strains (NBL 111, 113, 114, 117, 121) were subjected to the qualitative tests of Hosokawa (Stanier et al. 1966) to determine the pathways which they use for the dissimilation of benzoate and $p$-hydroxybenzoate. In every case, extracts prepared from organisms grown on these two compounds convert the respective diphenolic intermediates, catechol and protocatechuate, to $\beta$-ketoadipate. Accordingly, ring cleavage is of the ortho type, as in fluorescent pseudomonads and Pseudomonas multivorans (Stanier et al. 1966).

The same five strains were examined for constitutive synthesis of the arginine dihydrolase system, with positive results in every case. This is a biochemical property that is shared with the fluorescent pseudomonads, but not with Pseudomonas multivorans (Stanier et al. 1966).

As reported in the paper by Mandel (1966), the DNA samples isolated from 5 strains of Pseudomonas pseudomallei are of relatively high guanine + cytosine (GC) content, averaging 69 moles \% GC, with very little variation between samples. This value is at the upper limit of the range recorded for aerobic pseudomonads.

As shown in Tables 1-3, the range of organic compounds utilizable as sole sources of carbon and energy is exceptionally wide, and the nutritional patterns of the 26 strains examined are on the whole very homogeneous. No less than 80 of the compounds tested can be used by at least 24 strains, the great majority of them by all strains (Table 1). Pseudomonas pseudomallei accordingly ranks among the most nutritionally versatile members of the genus Pseudomonas. The list of utilizable compounds includes a large number of carbohydrates, notably fucose, D-arabinose, cellobiose, maltose and starch, all of which are rarely utilized by aerobic pseudomonads. Fatty acids and most dicarboxylic acids, as well as a variety of other organic acids, can support growth. Several aromatic compounds (benzoate, $p$-hydroxybenzoate, anthranilate, phenylacetate) are universally used. Eighteen amino acids, several higher amines and two $N$-methyl compounds are used. The utilizable amino acids include $L$-threonine, very rarely attacked by aerobic pseudomonads. However, the alcohols, which are used as substrates by most aerobic pseudomonads, are in general not substrates for $P$. pseudomallei. Only three strains (NBL 115, 118, 125) can grow on ethanol. Strain 115 can also grow on $n$-propanol, isopropanol, $n$-butanol isobutanol and ethyleneglycol; and strain 125 on the latter three compounds.

In the general character of its nutritional spectrum Pseudomonas pseudomallei resembles most closely the fluorescent pseudomonads and $\boldsymbol{P}$. multivorans, which are also nutritionally versatile groups. However, many characters serve to distinguish $P$. pseudomallei from these aerobic pseudomonads. Distinguishing characters vis-à-vis the whole group of fluorescent pseudomonads include: accumulation of poly- $\beta$-hydroxybutyric acid as a cellular reserve material; ability to use poly- $\beta$ hydroxybutyric acid as an exogenous carbon source; lack of fluorescent pigment 
production; utilization of D-arabinose, fucose, maltose, cellobiose and starch. Distinguishing characters vis-à-vis $\boldsymbol{P}$. multivorans include: ability to use poly$\beta$-hydroxybutyric acid as an exogenous carbon source; capacity for denitrification; constitutive production of arginine dihydrolase; growth on starch; failure to grow

Table 1. Pseudomonas pseudomallei. Compounds used as substrates by 23 or more strains

\begin{tabular}{|c|c|c|}
\hline Carbohydrates and & Hydroxyacids & $\beta$-Alanine \\
\hline sugar derivatives & L-Malate & L-Serine* \\
\hline D-Ribose & DL- $\beta$-Hydroxybutyrate & L-Threonine \\
\hline D-Arabinose & DL-Lactate & L-Isoleucine* \\
\hline D-Fucose & DL-Glycerate & L-Valine \\
\hline D-Glucose & Poly- $\beta$-Hydroxybutyrate & L-Aspartate \\
\hline D-Mannose & & L-Glutamate \\
\hline D-Galactose & Miscellaneous & L-Lysine* \\
\hline D-Fructose & organic acids & DL-Arginine \\
\hline Sucrose & Citrate & $\gamma$-Aminobutyrate \\
\hline Trehalose & $\alpha$-Ketoglutarate & $\delta$-Aminovalerate \\
\hline Maltose & Pyruvate & Amino acids and \\
\hline Cellobiose & Aconitate & related compounds \\
\hline $\begin{array}{l}\text { Salicin } \\
\text { Starch }\end{array}$ & Levulinate* & containing a ring \\
\hline $\begin{array}{l}\text { Starch } \\
\text { Gluconate }\end{array}$ & & structure \\
\hline 2-Ketogluconate & and glycols & L-Histidine \\
\hline Fatty acids & Erythritol & $\begin{array}{l}\text { L-Proline } \\
\text { L-Tyrosine }\end{array}$ \\
\hline Acetate & $\begin{array}{l}\text { Mannitol } \\
\text { Sorbitol }\end{array}$ & L-Phenylalanine \\
\hline Propionate & & L-Tryptophan \\
\hline Butyrate & $\begin{array}{l}\text { Inositol } \\
\text { Glycerol }\end{array}$ & L-Kynurenine \\
\hline Isobutyrate & & Kynurenate \\
\hline $\begin{array}{l}\text { Valerate } \\
\text { Isovalerate }\end{array}$ & Alcohols & Anthranilate* \\
\hline $\begin{array}{l}\text { Isovalerate } \\
\text { Caproate }\end{array}$ & (None) & Amines \\
\hline Heptanoate & Non-nitrogenous & Ethanolamine \\
\hline Caprylate & aromatic and other & Putreseine \\
\hline Pelargonate & cyclic compounds & Butylamine* \\
\hline Caprate & Benzoylformate & $\alpha$-Amylamine \\
\hline $\begin{array}{l}\text { Dicarboxylic } \\
\text { Acids }\end{array}$ & $\begin{array}{l}\text { Benzoate } \\
p \text {-Hydroxybenzoate } \\
\text { Phenylacetate }\end{array}$ & $\begin{array}{c}\text { Miscellaneous } \\
\text { nitrogenous compounds }\end{array}$ \\
\hline Succinate & Quinate & Betaine \\
\hline Fumarate & & Sarcosine* \\
\hline Adipate & Aliphatic amino & Hippurate \\
\hline $\begin{array}{l}\text { Suberate } \\
\text { Azelate* }\end{array}$ & acids & \\
\hline $\begin{array}{l}\text { Azelate } \\
\text { Sebacate }\end{array}$ & $\begin{array}{l}\text { L- } \alpha \text {-Alanine } \\
\text { D- } \alpha \text {-Alanine }\end{array}$ & $\begin{array}{l}\text { Paraffinic hydrocarbons } \\
\text { (None) }\end{array}$ \\
\hline
\end{tabular}

* Strains which fail to use this compound are shown in Table 3; all other compounds are universal substrates.

on 0 - and $m$-hydroxybenzoate, uracil and testosterone. The only other Pseudomonas species with which $\boldsymbol{P}$. pseudomallei shares some conspicuous traits is $\boldsymbol{P}$. stutzeri. Both these species can produce peculiar rough wrinkled colonies, quite unlike those of any other well-characterized member of the aerobic pseudomonads. Two other characters shared with $\boldsymbol{P}$. stutzeri are the ability to hydrolyse starch and to deni- 
trify. However, the two species can be very easily distinguished by morphological and physiological characters. The flagellation of $\boldsymbol{P}$. stutzeri is monotrichous, that of $P$. pseudomallei multitrichous (Leifson, 1960). $P$. stutzeri can neither accumulate poly- $\beta$-hydroxybutyric acid as a cellular reserve material nor use it as an exogenous carbon source, and it is much less versatile nutritionally than $\boldsymbol{P}$. pseudomallei.

\section{Table 2. Pseudomonas pseudomallei. Compounds not used by any strain}

Carbohydrates and
$\quad$ sugar derivatives
L-Arabinose
D-Xylose
L-Rhamnose
Lactose
Inulin
Saccharate
Mucate
$\quad$ Dicarboxylic
$\quad$ acids
Oxalate
Malonate
Maleate
$\quad$ Hydroxy acids
D-(-)-Tartrate
L-(+)-Tartrate
meso-Tartrate
$\quad$ Miscellaneous
$\quad$ organic acids
Citraconate
Itaconate
Mesaconate

\begin{tabular}{l} 
Polyalcohols \\
and glycols \\
Propyleneglycol \\
2,3-Butyleneglycol \\
\multicolumn{1}{c}{ Alcohols } \\
Methanol \\
Geraniol
\end{tabular}

Non-nitrogenous
aromatic and other
cyclic compounds
D-Mandelate
o-Hydroxybenzoate
m-Hydroxybenzoate
Phthalate
Isophthalate
Terephthalate
Phenylethanediol
Phenol
Testosterone

Aliphatic amino acids

Glycine

L-Norleucine

DL-Citrulline

DL- $\alpha$-Aminobutyrate

DL- $\alpha$-Aminovalerate

\author{
Amino acids and related \\ compounds containing \\ a ring structure \\ D-Tryptophan \\ $m$-Aminobenzoate \\ $p$-Aminobenzoate
}

\section{Amines}

Methylamine

Benzylamine

Tryptamine

$$
\begin{aligned}
& \text { Miscellaneous nitrogenous } \\
& \text { compounds } \\
& \text { Creatine } \\
& \text { Pantothenate } \\
& \text { Nicotinate } \\
& \text { Paraffinic hydrocarbons } \\
& n \text {-Dodecane }
\end{aligned}
$$

\section{Characterization of Bacillus mallei}

The 15 strains of Bacillus mallei examined constitute a reasonably uniform group in terms of the characters examined, although there was more internal variation than in Pseudomonas pseudomallei. The form and colour of growth of $\boldsymbol{B}$. mallei on solid media did not show the variations found with $\boldsymbol{P}$. pseudomallei. Colonies ranged from smooth cream to smooth white. An occasional rough white was seen with one of the strains.

Except as specifically noted, the following characters were shared by all strains. Poly- $\beta$-hydroxybutyric acid was accumulated as a cellular reserve material. There was no growth under strictly anaerobic conditions in a complex medium containing glucose. There was abundant growth under strictly anaerobic conditions in a complex medium containing nitrate; but only five strains (NBL 1, 2, 6, 7, 10) produced visible gas. Growth occurred at $42^{\circ}$ but not at $5^{\circ}$. We were unable to find any statements in the literature about the minimal growth requirements of Bacillus mallei. However, every strain grew in chemically defined media containing ammonia as the 
sole nitrogen source. The growth in such media was very slow; but even in complex media B. mallei grew relatively slowly, as compared with Pseudomonas pseudomallei. Gelatin and Tween 80 were hydrolysed by means of extracellular enzymes. Eight strains (NBL 1, 4, 5, 6, 7, 10, 16, 18) hydrolysed starch; and all but two strains (NBL 5,6$)$ hydrolysed poly- $\beta$-hydroxybutyric acid by extracellular enzymes.

Table 3. Pseudomonas pseudomallei. Substrates utilized by a fraction of the strains

\begin{tabular}{lcl} 
Substrate & $\begin{array}{c}\text { No. of } \\
\text { positive } \\
\text { strains }\end{array}$ & \multicolumn{1}{c}{ Negative strains } \\
Glutarate & 18 & $111,119,125,128,130,133,135,138$ \\
Pimelate & 18 & $101,103,111,119,121,122,125,128$ \\
Azelate & 23 & $111,117,119$ \\
D-Malate & 16 & $102,103,111,117,121,128,130,133,135,138$ \\
Levulinate & 24 & 119,121 \\
Adonitol & 14 & $102,103,111,112,113,114,115,118,121,130,133,135$ \\
Ethyleneglycol & 2 & All except 115, 125 \\
Ethanol & 3 & All except 115, 118, 125 \\
$n$-Propanol & 1 & All except 115 \\
Isopropanol & 1 & All except 115 \\
$n$-Butanol & 2 & All except 115, 125 \\
Isobutanol & 2 & All except 115, 125 \\
L-Mandelate & 18 & $111,115,117,119,120,125,137,138$ \\
$p$-Hydroxy- & 24 & 124,126 \\
benzoate & & \\
L-Serine & 25 & 118 \\
L-Leucine & 2 & All except 114, 121 \\
L-Isoleucine & 25 & 111 \\
L-Lysine & 25 & 104 \\
DL-Ornithine & 1 & All except 104 \\
Anthranilate & 25 & 128 \\
Spermine & 18 & $103,111,117,128,130,133,135,138$ \\
Butylamine & 24 & 103,121 \\
$\alpha$-Amylamine & 24 & 103,121 \\
Sarcosine & 23 & $103,111,121$ \\
Trigonelline & 10 & All except 101, 111, 115, 117, 119, 122, 130, 135, 137, 138 \\
$n$-Hexadecane & 4 & All except 117, 118, 135, 137 \\
& &
\end{tabular}

All strains, except NBL 1, produce a constitutive arginine dihydrolase. All strains except NBL 4 and 12, cleave protocatechuate and catechol by ortho oxidation.

As shown by Mandel (1966) the DNA of Bacillus mallei contains $69 \%$ GC, a value identical with that for Pseudomonas pseudomallei.

The spectrum of organic compounds utilizable by Bacillus mallei as sole sources of carbon and energy resembles that of $\boldsymbol{P}$. pseudomallei, but is considerably less extensive, and also more variable from strain to strain (Tables 4-6). A total of 46 different organic compounds was used by at least 13 of the 15 strains examined; most of them were used by all 15 strains (Table 4). The list includes a constellation of compounds which were also attacked by $\boldsymbol{P}$. pseudomallei, but rarely by other aerobic pseudomonads : fucose, $\mathrm{D}$-arabinose, cellobiose, L-threonine, poly- $\beta$-hydroxybutyric acid. Maltose and starch, universally used by $\boldsymbol{P}$. pseudomallei, were used by 8 out of 15 strains of Bacillus mallei.

In Table 7 we have summarized the principal nutritional differences between Bacillus mallei and Pseudomonas pseudomallei. Only four compounds which were 
wholly non-utilizable by $P$. pseudomalle $i$ were attacked by $B$. mallei: D-xylose and glycine (used by 14 strains); DL- $\alpha$-aminobutyrate (used by 13 strains); L-arabinose (used by 8 strains). Growth on $\mathrm{D}$-xylose, on glycine and on $\alpha$-aminobutyrate are, accordingly, the only positive nutritional characters that clearly distinguish $B$. mallei from $P$. pseudomallei. On the other hand, no less than 18 compounds which

\section{Table 4. Bacillus mallei. Compounds used as substrates by 13 or more strains}

Carbohydrates and
sugar derivatives
D-Xylose*
D-Arabinose*
D-Fucose
D-Glucose
D-Mannose
D-Galactose
Sucrose
Trehalose
Cellobiose
Gluconate*

Fatty acids

Acetate

Propionate*

Dicarboxylic acids

Malonate*

Succinate*

Fumarate

Adipate*

$\quad$ Hydroxy-acids
L-Malate
DL- $\beta$-Hydroxybutyrate
DL-Lactate
DL-Glycerate*
Poly- $\beta$-Hydroxybutyrate

$$
\begin{gathered}
\text { Miscellaneous organic } \\
\text { acids }
\end{gathered}
$$

$\alpha$-Ketoglutarate

Pyruvate*

Polyalcohols and glycols

Mannitol
Sorbitol*
Inositol*
Glycerol

Alcohols

(None)

Non-nitrogenous aromatic and other cyclic compounds

Benzoate

p-Hydroxybenzoate

Phenylacetate

Quinate*

Aliphatic amino
acids
Glycine*
L- $\alpha$-Alanine
D- $\alpha$-Alanine

\author{
$\beta$-Alanine* \\ L-Threonine \\ L-Aspartate* \\ L-Glutamate \\ DL-Arginine \\ DL- $\alpha$-Aminobutyrate* \\ $\gamma$-Aminobutyrate
}
Amino acids and related compounds containing a ring structure
L-Histidine
L-Tyrosine
L-Tryptophan

Amines
(None)
Miscellaneous nitrogenous
compounds
Betaine*
Hippurate
Paraffinic hydrocarbons
(None)

* Strains which fail to use this compound are shown in Table 6; all others are universal substrates.

did not support growth of any strain of $B$. mallei were used by all or the great majority of strains of $\boldsymbol{P}$. pseudomallei. As shown in Table 7 , the compounds in question are chemically diverse, and include carbohydrates, fatty acids, aromatic compounds, amino acids and amines. Accordingly, it is evident that the specific nutritional differences between $B$. mallei and $P$. pseudomallei are overwhelmingly represented by characters for which $B$. malle $i$ strains are negative; $B$. malle $i$ clearly lacks many enzymic potentialities possessed by $\boldsymbol{P}$. pseudomallei.

Nine compounds were more or less consistently utilized by some strains of Bacillus mallei but not by others (Table 8 ). With the exception of L-arabinose, they are all substrates for Pseudomonas pseudomallei, so that strains of $B$. malle $i$ which utilized them resemble $\boldsymbol{P}$. pseudomallei more closely in nutritional spectrum than those which do not. These nutritional differences might appear to justify the recognition of two distinct varieties of $B$. mallei, were it not for an interesting feature 


\section{M. S. Redfearn, N. J. Palleroni and R. Y. Stanier}

of the strain histories. Strains 7, 10 and 11 are all derived from a single source, a human infection in China. Strains 7 and $\mathbf{1 0}$ have been maintained in culture or in lyophil preparation since their isolation in 1944 and are nutritionally identical; they can use all nine compounds listed in Table 8. Strain 10 was subjected, some years after its primary isolation, to three serial passages in the hamster; the strain re-

\section{Table 5. Bacillus mallei. Compounds not utilized by any of the strains}

Carbohydrates and
sugar derivatives
D-Ribose
L-Rhamnose
Lactose
Inulin
Saccharate
Mucate

Fatty acids

Isobutyrate

Valerate

Isovalerate

Caproate

Heptanoate

Pelargonate

Caprate
Dicarboxylic acids

Oxalate

Maleate

Eicosanedioate

$$
\text { Hydroxy-acids }
$$

D-( -)-Tartrate

L-(+)-Tartrate

meso-Tartrate

Glycollate

Hydroxymethylglutarate

\section{Miscellaneous}

organic acids

Levulinate

Citraconate

Itaconate

Mesaconate

\author{
Polyalcohols and \\ glycols \\ Erythritol \\ Ethyleneglycol \\ Propyleneglycol \\ 2,3-Butyleneglycol
}

Alcohols

Methanol

Ethanol

n-Propanol

Isopropanol

n-Butanol

Isobutanol

Geraniol

Non-nitrogenous aromatic and other cyclic compounds

D-Mandelate

L-Mandelate

Benzoylformate

o-Hydroxybenzoate

$m$-Hydroxybenzoate

Phthalate

Isophthalate

Terephthalate

Phenylethanediol

Phenol

Testosterone

$$
\begin{gathered}
\text { Aliphatic amino } \\
\text { acids }
\end{gathered}
$$

L-Leucine

L-Isoleucine

L-Norleucine

L-Lysine

DL-Ornithine

DL-Citrulline

DL- $\alpha$-Aminovalerate

\author{
Amino acids and related \\ compounds containing \\ a ring structure \\ D-Tryptophan \\ L-Kynurenine \\ Kynurenate \\ m-Aminobenzoate \\ p-Aminobenzoate
}

Amines

Methylamine

Ethanolamine

Benzylamine

Spermine

Histamine

Tryptamine

Butylamine

$\alpha$-Amylamine

\begin{tabular}{l}
\multicolumn{1}{c}{ Miscellaneous } \\
nitrogenous compounds \\
Creatine \\
Pantothenate \\
Acetamide \\
Nicotinate \\
Paraffinic hydrocarbons \\
n-Dodecane \\
$n$-Hexadecane
\end{tabular}

isolated after these passages is strain 11. Strain 11 can use only two of the compounds listed in Table 8; furthermore, it cannot grow on either sorbitol or aspartate, both of which support the growth of strains 7 and 10. The reliability of strain histories cannot, of course, be taken for granted; but these facts do suggest that the nutritional characters of $\boldsymbol{B}$. mallei may be modifiable by animal passage. Until this possibility has been further studied, the taxonomic significance of the data in Table 8 cannot be assessed. 


\section{Proposed neotype strains}

The original isolates of Bacillus mallei and Pseudomonas pseudomallei have long since been lost. Lysenko (1961) proposed a neotype strain for $\boldsymbol{P}$. pseudomallei; but the properties of this strain differ in many respects from those of the strains which

Table 6. Bacillus mallei. Substrates utilized by a fraction of the strains

\begin{tabular}{|c|c|c|}
\hline Substrate & $\begin{array}{l}\text { No. of } \\
\text { positive } \\
\text { strains }\end{array}$ & Negative strains \\
\hline D-Xylose & 14 & 4 \\
\hline D-Arabinose & 14 & 4 \\
\hline L-Arabinose & 8 & $2,8,11,12,17,19,20$ \\
\hline D-Fructose & 9 & $2,8,12,17,19,20$ \\
\hline Maltose & 8 & $2,8,11,12,17,19,20$ \\
\hline Salicin & 6 & $1,2,4,6,8,12,17,19,20$ \\
\hline Starch & 8 & $2,8,11,12,17,19,20$ \\
\hline Gluconate & 13 & 4,20 \\
\hline 2-Ketogluconate & 11 & $1,2,4,20$ \\
\hline Propionate & 14 & 1 \\
\hline Butyrate & 11 & $1,8,17,19$ \\
\hline Caprylate & 8 & $1,8,11,12,17,19,20$ \\
\hline Malonate & 13 & 2,4 \\
\hline Succinate & 13 & 8,12 \\
\hline Glutarate & 3 & All except 8, 18, 19 \\
\hline Adipate & 13 & 4,19 \\
\hline Pimelate & 4 & All except 1, 5, 6, 8 \\
\hline Suberate & 3 & All except 1, 5, 6 \\
\hline Azelate & 2 & All except 1,5 \\
\hline Sebacate & 1 & All except 4 \\
\hline D-Malate & 10 & $4,8,12,19,20$ \\
\hline DL-Glycerate & 13 & 1,17 \\
\hline Poly- $\beta$-hydroxybutyrate & 13 & 5,6 \\
\hline Citrate & 10 & $8,11,12,17,19$ \\
\hline Pyruvate & 13 & 8,19 \\
\hline Aconitate & 1 & All except 1 \\
\hline Sorbitol & 14 & 11 \\
\hline Inositol & 14 & 8 \\
\hline Adonitol & 1 & All except 7 \\
\hline Quinate & 14 & 8 \\
\hline Glycine & 14 & 2 \\
\hline$\beta$-Alanine & 14 & 4 \\
\hline L-Serine & 3 & All except $16,18,20$ \\
\hline $\mathrm{L}$-Valine & 9 & $1,4,12,17,19,20$ \\
\hline L-Aspartate & 14 & 11 \\
\hline DL- $\alpha$-Aminobutyrate & 13 & 1,12 \\
\hline$\delta$-Aminovalerate & 9 & $5,11,12,17,19,20$ \\
\hline L-Proline & 8 & $1,8,11,12,17,19,20$ \\
\hline L-Phenylalanine & 9 & $4,8,12,17,19,20$ \\
\hline Anthranilate & 11 & $2,4,6,10$ \\
\hline Putrescine & 6 & $1,2,4,6,8,12,17,19,20$ \\
\hline Betaine & 14 & 4 \\
\hline Sarcosine & 8 & $1,2,4,8,12,19,20$ \\
\hline Trigonelline & 8 & $1,8,11,12,17,19,20$ \\
\hline
\end{tabular}

we have examined. Furthermore, the description of Lysenko's strain in the catalogue of the American Type Culture Collection (1964) carries the following comment: 'Pseudomonas aeruginosa (W. C. Haynes, personal communication).' Hence it seems 


\section{M. S. Redfearn, N. J. Palleroni and R. Y. Stanier}

necessary to designate another neotype. We propose NBL 121 as the neotype"strain of $\boldsymbol{P}$. pseudomallei. Apparently no neotype strain of $\boldsymbol{B}$. mallei has been designated; we propose NBL 7 .

'Table 7. Principal nutritional differences between

Pseudomonas pseudomallei and Bacillus mallei

\begin{tabular}{lcc} 
& \multicolumn{2}{c}{ Fraction of positive strains } \\
\cline { 2 - 3 } \multicolumn{1}{c}{ Compound } & P. pseudomallei & $P$. mallei \\
D-Ribose & $26 / 26$ & $0 / 15$ \\
D-Xylose & $0 / 26$ & $14 / 15$ \\
L-Arabinose & $0 / 26$ & $8 / 15$ \\
Isobutyrate & $26 / 26$ & $0 / 15$ \\
Valerate & $26 / 26$ & $0 / 15$ \\
Isovalerate & $26 / 26$ & $0 / 15$ \\
Caproate & $26 / 26$ & $0 / 15$ \\
Heptanoate & $26 / 26$ & $0 / 15$ \\
Pelargonate & $26 / 26$ & $0 / 15$ \\
Caprate & $26 / 26$ & $0 / 15$ \\
Levulinate & $24 / 26$ & $0 / 15$ \\
Erythritol & $26 / 26$ & $0 / 15$ \\
Glycine & $0 / 26$ & $14 / 15$ \\
L-Isoleucine & $25 / 26$ & $0 / 15$ \\
L-Lysine & $25 / 26$ & $0 / 15$ \\
DL- $\alpha$-Aminobutyrate & $0 / 26$ & $13 / 15$ \\
L-Kynurenine & $26 / 26$ & $0 / 15$ \\
Kynurenate & $26 / 26$ & $0 / 15$ \\
Ethanolamine & $26 / 26$ & $0 / 15$ \\
Butylamine & $24 / 26$ & $0 / 15$ \\
a-Amylamine & $24 / 26$ & $0 / 15$ \\
& &
\end{tabular}

Table 8. Differences in nutritional spectrum between two groups of strains of Bacillus mallei, together with the corresponding nutritional properties of Pseudomonas pseudomallei

\begin{tabular}{|c|c|c|c|c|c|c|c|c|c|c|c|c|c|c|c|c|}
\hline \multirow[b]{2}{*}{ Compound } & \multicolumn{15}{|c|}{ Strains of $B$. malle $i$} & \multirow{2}{*}{$\begin{array}{l}P . \text { pseudo- } \\
\text { mallei: } \\
\text { fraction of } \\
\text { positive } \\
\text { strains }\end{array}$} \\
\hline & 8 & 11 & 12 & 17 & 19 & 20 & 1 & $\mathbf{2}$ & 4 & 5 & 6 & 7 & 10 & 16 & 18 & \\
\hline L-Arabinose & - & - & - & - & - & - & + & - & + & + & + & + & + & + & + & $0 / \mathbf{2 6}$ \\
\hline Maltose & - & - & - & - & - & - & + & - & + & + & + & + & + & + & + & $26 / 26$ \\
\hline Starch & - & - & - & - & - & - & + & - & + & + & + & + & + & + & + & $26 / 26$ \\
\hline Caprylate & - & - & - & - & - & - & - & + & + & + & + & + & + & + & + & $26 / 26$ \\
\hline Citrate & - & - & - & - & - & - & + & + & + & + & + & + & + & + & + & $26 / 26$ \\
\hline L-Phenylalanine & - & + & - & - & - & - & + & + & - & + & + & + & + & + & + & $26 / 26$ \\
\hline Trigonelline & - & - & - & - & - & - & - & + & + & + & + & + & + & + & + & $26 / 26$ \\
\hline L-Proline & - & - & - & - & - & - & - & + & + & + & + & + & + & + & + & $26 / 26$ \\
\hline Sarcosine & - & + & - & - & - & - & - & - & - & + & + & + & + & + & + & $23 / 26$ \\
\hline
\end{tabular}

\section{DISCUSSION}

The affinities and taxonomic position of Pseudomonas pseudomallei and Bacillus mallei

Our characterization of Pseudomonas pseudomallei and Bacillus mallei in terms of physiological and nutritional properties amply confirms the close resemblance between these two species, which has been suggested by others on the basis of simi- 
larities in pathogenicity (Whitmore, 1913), antigenic constitution (Stanton \& Fletcher, 1925; Cravitz \& Miller, 1950) and phage susceptibility (Smith \& Cherry, 1957). The phenotypic resemblances assume even deeper significance in view of the virtual identity in the GC content of the respective DNAs (Mandel, 1966). The natural affinity between these two bacterial species seems now established beyond reasonable doubt; consequently, the maintenance of a generic separation between them seems unnecessary and undesirable.

At the same time, the present characterization of Pseudomonas pseudomalleithe first which can be considered reasonably complete-fully supports its current assignment to the genus $\boldsymbol{P s e u d o m o n a s . ~} \boldsymbol{P}$. pseudomallei conforms in every respect to the generic definition proposed by Stanier et al. (1966). This being so, we cannot avoid the conclusion that Bacillus mallei should also be placed in the genus Pseudomonas, despite its permanent non-motility. We therefore proposed to designate it, as $\boldsymbol{P s e u d o m o n a s ~ m a l l e i , ~ c o m b . n o v . ~}$

Polar flagellation has heretofore been considered a cardinal criterion for membership in the genus Pseudomonas. It is true that Jessen (1965) has described rare strains of the type species, Pseudomonas aeruginosa, which appear to be permanently non-motile; but polar flagellation is characteristic of the overwhelming majority of strains which belong to this species. No strain of Pseudomonas mallei has ever been reported to show motility, and the presumption is therefore strong that this species is completely non-motile.

In other groups of Gram-negative polarly-flagellate bacteria, non-motile species have long been associated with motile ones, though generally not within the confines of a single genus. Among photosynthetic bacteria, many species of purple sulphur bacteria are non-motile; and green bacteria are characteristically nonmotile, only one species with polar flagella having been described to date. The taxonomic association of polarly flagellate and non-motile species among photosynthetic bacteria has never been questioned by bacterial taxonomists, who have more or less tacitly assumed in this case that the shared physiological and biochemical properties afford an overwhelming presumption of evolutionary affinity. The same line of reasoning justifies the taxonomic association that we now propose between Pseudomonas pseudomallei and $\boldsymbol{P}$. mallei.

However, the abandonment of polar flagellation as an essential attribute of the genus Pseudomonas does entail some practical disadvantages. It opens the generic portals to a large number of species of non-motile Gram-negative rod-shaped eubacteria now allotted to such ill-defined genera as Flavobacterium and Achromobacter. The characterization of most of these species is grossly inadequate. Hence, as a matter of practical policy, we believe that they should be declared "passportless cosmopolitans' until they have been shown to conform to all the criteria proposed by Stanier et al. (1966) for the genus Pseudomonas, except for the possession of flagella. If any species can pass this test, we see no valid grounds for denying it admission to $\boldsymbol{P}$ seudomonas. It should be recognized that the taxonomic problem raised here has always been immanent in schemes of eubacterial classification which use the mode of flagellation as a major differential character, since such schemes by their very nature leave the many permanently non-motile species in an indeterminate state.

The phenotypic resemblances between Pseudomonas pseudomallei and P. mallei 
are so great that these two species can be regarded as constituting a generic subgroup, analogous to the fluorescent, acidovorans and alcaligenes groups described by Stanier et al. (1966). For purposes of differentiating the 'pseudomallei group' as we shall term it, from other aerobic pseudomonads, we have constructed an ideal group phenotype, comprising a total of 16 unrelated characters which are shared by most strains of $P$. pseudomallei and $P$. mallei (Table 9). In Table 10 we have calculated the degree of conformity of all species of the genus Pseudomonas which have

Table 9. The group of characters of the greatest value in the differentiation of Pseudomonas pseudomallei and $\boldsymbol{P}$. mallei from other aerobic pseudomonads

\begin{tabular}{|c|c|c|c|}
\hline \multirow[b]{2}{*}{ Characters } & \multicolumn{2}{|c|}{ No. of positive strains } & \multirow{2}{*}{$\begin{array}{l}\text { Ideal } \\
\text { phenotype for } \\
\text { the group }\end{array}$} \\
\hline & $\begin{array}{c}P . \\
\text { pseudomallei }\end{array}$ & P. mallei & \\
\hline $\begin{array}{l}\text { Poly- } \beta \text {-hydroxybutyrate } \\
\text { as reserve material }\end{array}$ & 26 & 15 & + \\
\hline $\begin{array}{l}\text { Poly- } \beta \text {-hydroxybutyrate } \\
\text { degradation by extra- } \\
\text { cellular enzymes }\end{array}$ & 26 & 13 & + \\
\hline Denitrification & $\mathbf{2 6}$ & 15 & + \\
\hline Fluorescent pigment & 0 & 0 & - \\
\hline \multicolumn{4}{|l|}{ Utilization of: } \\
\hline Starch & 26 & 8 & + \\
\hline Cellobiose & 26 & 15 & + \\
\hline D-Fucose & 26 & 15 & + \\
\hline D-Arabinose & 26 & 14 & + \\
\hline Adipate & 26 & 13 & + \\
\hline Mucate & $\mathbf{0}$ & 0 & - \\
\hline$m$-Hydroxybenzoate & $\mathbf{0}$ & 0 & - \\
\hline$p$-Hydroxybenzoate & $\mathbf{2 4}$ & 15 & + \\
\hline DL-Arginine & 26 & 15 & + \\
\hline L-Threonine & 26 & 15 & + \\
\hline Betaine & 26 & 14 & + \\
\hline Testosterone & 0 & 0 & - \\
\hline
\end{tabular}

been characterized on the basis of the methods here used to the ideal phenotype of the pseudomallei group. Of the $\mathbf{4 1}$ strains of the pseudomallei group that we have examined, 39 either conform perfectly to the ideal phenotype, or deviate by a single character; one strain of $\boldsymbol{P}$. mallei deviates by two characters; and one, by three. Among other Pseudomonas species, $\boldsymbol{P}$. multivorans most closely approaches the ideal phenotype of the pseudomallei group, all 19 strains of $P$. multivorans deviating from it by 6 characters. In all remaining Pseudomonas species, no strain deviates by less than 8 characters.

The existence of a close relationship between Pseudomonas pseudomallei and $\boldsymbol{P}$. aeruginosa has been repeatedly suggested by earlier workers. This suggestion stemmed in part from epidemiological and pathological resemblances; and it is true that in these respects there are some interesting parallels to be discussed. On the basis of a comparative study Wetmore \& Gochenour (1956) concluded that the resemblances extend to cultural and physiological characters, and alleged that it is difficult to distinguish achromogenic strains of $\boldsymbol{P}$. aeruginosa from $\boldsymbol{P}$. pseudomallei. In reality, as Table 10 shows, the differences between the two species are numerous. 
Of the 16 characters selected to define the ideal phenotype of the pseudomallei group, no strain of $\boldsymbol{P}$. pseudomallei possesses less than 15; and no strain of $\boldsymbol{P}$. aeruginosa more than 8 . Hence even on this restricted set of traits, the two species are readily differentiable. (The total number of phenotypic traits by which they differ is, of course, much greater. We have not precisely determined it; but this can be readily done by the numerically inclined taxonomist, by using the complete data for Pseudomonas aeruginosa (Stanier et al. 1966) and the complete data given here for $\boldsymbol{P}$. pseudomallei).

The conclusion reached by Wetmore \& Gochenour is evidently incorrect but instructive. It shows how far astray the bacterial taxonomist can be led if he uses a set of 'standard methods' which are either inadequate or inappropriate for the particular bacterial group to which he applies them.

Table 10. Conformity of strains of different species of aerobic pseudomonads to the selected 16 characters (Table 9) which define the ideal phenotype of the pseudomallei group

Total no. of characters shared with ideal phenotype

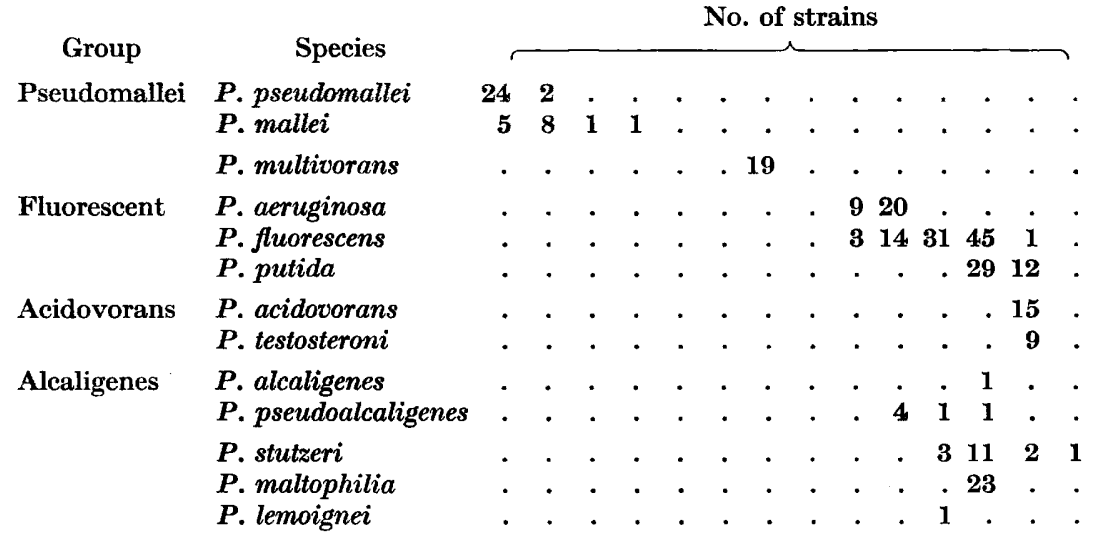

\section{Ecological and evolutionary considerations}

As a pathogen Pseudomonas pseudomallei presents many ecological puzzles. Nigg (1963) summed up half a century of work on this subject in one sentence: 'almost nothing is known about the epidemiology of melioidosis, but textbooks state that rodents constitute the natural reservoir of infection'. We shall now attempt to construct a coherent ecological hypothesis, which takes into account the existing information about the pathogenic behaviour and epidemiology of $\boldsymbol{P}$. pseudomallei and our present findings about its physiological properties. $P$. pseudomalle $i$ shows an exceptionally low degree of host specificity. Natural infections have been reported in man, horse, cow, pig, sheep, goat, cat, dog and several different rodents. This wide host spectrum has been confirmed and extended by the experimental studies of Miller et al. (1948). The clinical and pathological manifestations of melioidosis are extremely varied. There are no distinctive symptoms, and correct diagnosis is essentially dependent on the isolation of the causative organism. 
Once melioidosis has become clinically recognizable, it typically follows a progressive course, and the mortality is very high. However, there are many indications that $P$ seudomonas pseudomallei cannot easily establish itself in the mammalian host. Human melioidosis is a relatively rare disease, even in south-east Asia where its incidence is highest. No case of contact infection has been reported in man, although patients with the disease have often not been isolated. Stanton \& Fletcher (1925) stated: ' $m a n$ is not readily susceptible; otherwise cases of melioidosis would surely have occurred in laboratories where living cultures have been freely handled or among the Asiatic attendants who have looked after hundreds of infected animals' (sic).

Clinical findings are relevant in this connexion. Roques \& Dauphin (1943) and Alain, Saint-Etienne \& Reynes (1949) observed that melioidosis may develop secondarily in patients hospitalized for other reasons, including surgical treatment and non-infectious disease. The detailed histories of the first six human cases recorded in northern Australia (Rimington, 1962) reveal the interesting fact that five of the affected individuals also suffered from chronic debilitating diseases. Melioidosis is evidently apt to develop in individuals with an unusually low degree of resistance. Nigg (1963) showed by serological tests that about $10 \%$ of a group of over 500 healthy adult Thai males had been exposed to Pseudomonas pseudomallei. This finding also indicates that exposure is rarely followed by a clinically recognizable infection. Taken together, these pathological and epidemiological facts all lead to one conclusion: $P$. pseudomallei does not have the biological profile of a successful parasite. It has little invasive power, and its host and tissue specificity alike are unusually low. To put the matter another way, there is no evidence that $\boldsymbol{P}$. pseudomalle $i$ has undergone an evolutionary adaptation to parasitic existence through long participation in a host-parasite relationship.

There has been much speculation about the natural reservoir of Pseudomonas pseudomallei in south-east Asia. A reservoir in rats, suggested by Whitmore (1913) without evidence, has been overwhelmingly disproved by subsequent work. At the Institut Pasteur in Saigon (an area of high incidence of melioidosis), over 20,000 autopsies on rats, several thousand of which were accompanied by blood cultures, resulted in only one isolation of $\boldsymbol{P}$. pseudomallei (Alain et al. 1949). There is, on the other hand, evidence for the occurrence of $\boldsymbol{P}$. pseudomallei in soil and water in south-east Asia. Chambon (1955) subjected 150 samples of mud and water to bacteriological analysis, and was able to isolate $\boldsymbol{P}$. pseudomallei directly from five of them. Nigg \& Johnston (1961) emphasized in this connexion the relevance of an epidemiological observation. Three cases of acute melioidosis have occurred as a sequel to car accidents, in which the victim suffered lacerations that were heavily contaminated with muddy water.

The biological character of Pseudomonas pseudomallei as it has emerged from our work is completely in accord with the assumption that this bacterium is a normal inhabitant of soil and water. Its physiological properties, like those of other nutritionally versatile members of the genus (the fluorescent pseudomonads, the acidovorans group, Pseudomonas multivorans), potentially equip it to play many roles in the aerobic mineralization of organic matter, and in denitrification. The crucial evidence still lacking is the demonstration that $\boldsymbol{P}$. pseudomalle $i$ can be isolated from soil or water by specific enrichment methods. Nevertheless, we consider the pre- 
sumptive evidence that $\boldsymbol{P}$. pseudomalle $i$ exists in nature as a free-living organism to be strong. In this event, it must be regarded as an accidental pathogen that can on rare occasions find a niche in the animal body, either as a result of abnormally low host resistance, or because exceptionally favourable portals of entry have been created by physical trauma.

The ecology of the other typical Pseudomonas species pathogenic for warmblooded animals, $P$. aeruginosa, presents striking analogies. Pseudomonas aeruginosa is a common inhabitant of soil, and has been shown by specific enrichment experiments

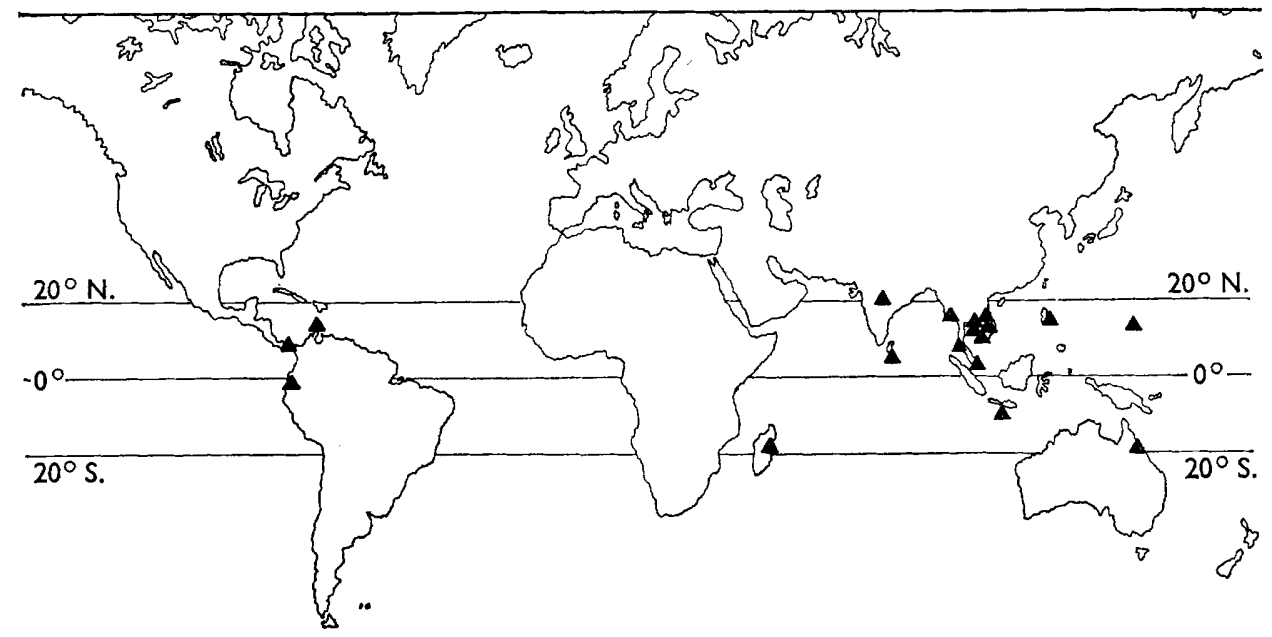

Fig. 1. Known geographical distribution of melioidosis. Each triangle indicates an area from which at least one bacteriologically diagnosed case of the disease has been reported.

to play a role in denitrification (Beijerinck \& Minkman, 1910) and in the oxidation of normal alkanes (Konovaltschikoff-Mazoyer \& Senez, 1956). Although it has assumed increasing clinical importance in recent decades as a result of its resistance to antibiotic therapy, it also does not have the profile of a successful parasite (Forkner, 1960). Laboratory infections have not been reported; the organism never gives rise to contact infections; and it is typically a hospital pathogen which establishes systemic infections in individuals who, for any one of a variety of medical reasons, have severely lowered degrees of resistance. Successful lodgement classically occurs when extensive artificial portals of entry have been created as a result of burns. Like $\boldsymbol{P}$. pseudomallei, $\boldsymbol{P}$. aeruginosa can be interpreted as a free-living organism which can occasionally develop in the animal body, but has not undergone any evolutionary selection for this rarely used capacity. It is no doubt significant that $\boldsymbol{P}$. pseudomallei and $\boldsymbol{P}$. aeruginosa invariably grow well at $41-42^{\circ}$. Growth at this temperature is irregular in $\boldsymbol{P}$. multivorans and $\boldsymbol{P}$. stutzeri, and fails wholly in all other Pseudomonas species except the alcaligenes group, of which too few strains have been examined to draw final conclusions on the temperature range (Stanier et al. 1966). The capacity to grow well around $40^{\circ}$ is obviously an essential precondition for occasional development in mammalian hosts.

However, Pseudomonas pseudomallei and P. aeruginosa do differ in one major ecological respect. Whereas $\boldsymbol{P}$. aeruginosa, like most free-living bacteria, has a world- 
wide distribution, $P$. pseudomallei apparently has not. It must be realized that the geographical distribution of the latter species is now exclusively inferred from its pathological manifestations, which almost certainly give a much too limited picture; but even taking this limitation into account, the facts are striking enough. The overwhelming majority of recorded cases of melioidosis both in man and in other animals have occurred in south-east Asia (Burma, Thailand, Malaya, Indochina, Indonesia). However, sporadic cases have been reported in other tropical areas, some far distant from the major focus. These include: Ceylon, Madagascar, northern Australia, the Caribbean region and Ecuador.* All these sites lie between $20^{\circ} \mathrm{N}$. and $20^{\circ} \mathrm{S}$. (Fig. 1). Hence it seems fair to assume that $\boldsymbol{P}$. pseudomallei is widely distributed through the tropical regions of the earth; and there is no epidemiological evidence for its occurrence outside them. Believing as we do that $P$. pseudomalle $i$ is a free-living organism, we are none the less forced to conclude that it has a limited geographical range, and occurs rarely, if at all, in temperate regions. A systematic study of its geographical ecology will be possible only when a method for its selective isolation from soil and water is devised. Our data on its physiological properties offer many indications of how this might be achieved.

In ecological terms, $P$ seudomonas mallei provides a striking contrast to $P$. pseudomallei and to $\boldsymbol{P}$. aeruginosa. The disease which it causes is almost entirely confined to horses, donkeys and mules, although infection is transmissible from equine hosts to man. Other animal species are susceptible, but do not seem to contract the disease in nature. In the infected animal, the infection can assume two sharply defined clinical forms: glanders, in which the primary focus of infection is the lungs; and farcy, in which the infection spreads (generally from a primary skin lesion) through the lymphatic system (Topley \& Wilson's Principles, 1955). These authors state: 'several cases of glanders have been reported in laboratory workers ; indeed, probably no organism, with the possible exception of Brucella tularensis, is so dangerous to work with as the glanders bacillus'.

Glanders is a disease that has been effectively eradicated from many European countries, Canada and the United States by the systematic destruction of infected animals and the application of quarantine measures. The remarkable success of these control programmes indicates that soil and water do not serve as reservoirs for Pseudomonas mallei, which can apparently exist in nature only through passage in susceptible animals. Its geographical range is therefore co-terminous with that of its natural hosts. In summary, $P$. malle $i$ is a successful parasite which shows evidence of long participation in a specific host-parasite relationship, to which it has become highly adapted. We consider it to be the only parasite of animals in the genus Pseudomonas.

Viewed in this context, the specific differences between Pseudomonas pseudomallei and $\boldsymbol{P}$. mallei, so remarkably similar in their general phenotypes, become highly

\footnotetext{
* Melioidosis is probably far more prevalent in many of these areas than the published reports suggest. The history of the disease in northern Australia (Rimington, 1962) throws an interesting light on this question. The first cases were discovered in 1949 in sheep. The first human case was diagnosed in 1950; and the report on it notes that identification of Pseudomonas pseudomallei, isolated from blood samples, was made at the local Animal Health Station. Subsequent outbreaks in a variety of domestic animals made it evident that melioidosis was endemic in the area; and in 1959-60, no less than five additional human cases were diagnosed. Rimington (1962), in discussing these facts, suggests that melioidosis may account for 'some of the undiagnosed fevers that occur in North Queensland'.
} 
significant. With minor exceptions, the characters by which they differ are negative in P. mallei. P. mallei lacks flagella; it has a much narrower range of carbon and energy sources; and it grows more slowly on artificial media, both chemically defined and complex. If we accept a common evolutionary origin for the two species, an assumption which is difficult to avoid, nearly all the specific structural and physiological differences between them can be interpreted as losses or impairments of function which took place in the $\boldsymbol{P}$. mallei line during the course of its transition from existence in soil and water to obligate dependence on a mammalian host. Equivalent functional regression has not taken place in the $\boldsymbol{P}$. pseudomallei line, because this bacterium (despite its pathogenic potentialities) has never succeeded in establishing a durable host-parasite relationship, and is consequently subject to evolutionary selection exclusively in the context of its activities as a free-living organism. Indeed, these two bacteria may provide an exquisite specific illustration of the evolutionary postulate of Lwoff (1944): adaptation to a parasitic mode of existence is typically accompanied by functional as well as structural regression.

This work was sponsored by the Office of Naval Research and the Bureau of Medicine and Surgery, United States Navy, under a contract between the Office of Naval Research and the Regents of the University of California.

\section{REFERENCES}

Alain, M., Saint-Etienne, J. \& Reynes, V. (1949). La mélioidose; considérations étiologiques, cliniques et pathogèniques à propos de 28 cas. Med. trop. 9, 191.

American Type Culture Collection, Catalogue of Cultures (1964). 7th ed., p. 88. Rockville, Maryland, U.S.A.

BeiJerinck, M. W. \& Minkman, D. C. J. (1910). Bildung und Verbrauch von Stickoxydul durch Bakterien. Zentbl. Bakt.ParasitKde (Abt. 2), 25, 30.

Bregeleisen, J. Z., Mosquera, R. \& Cherry, W. B. (1964). A case of human melioidosis: clinical, epidemiological and laboratory findings. Am. J. Trop. Med. Hyg. 13, 89.

Bokman, A. H., Levine, H. B. \& Lusby, M. (1957). Glucose catabolism in Malleomyces pseudomallei. J. Bact. 73, 649.

Brindle, C. S. \& Cowan, S. T. (1951). Flagellation and taxonomy of Whitmore's bacillus. J. Path. Bact. 63, 571.

Brumpt, E. (1910). Précis de Parasitologie, 1st ed. Paris: Masson.

Buchanan, R. E. (1918). Studies in the nomenclature and classification of the bacteria. V. Subgroups and genera of the Bacteriaceae. J. Bact. 3. 27.

Chambon, L. (1955). Isolement du bacille de Whitmore à partir du milieu extérieur. Ann. Inst. Pasteur, 89, 229.

Cotrew, G. S. (1950). Melioidosis in sheep in Queensland. A description of the causal organism. Aust. J. exp. Biol. med. Sci. 28, 676.

Cravitz, L. \& Mrleer, W. R. (1950). Immunologic studies with Malleomyces mallei and Malleomyces pseudomallei. I. Serological relationships between M. mallei and M. pseudomallei. J. infect. Dis. 86, 46.

Davie, J. \& Wells, C. W. (1952). Equine melioidosis in Malaya. Br. vet. J. 108, 161.

Entner, N. \& Doudoroff, M. (1952). Glucose and gluconic acid oxidation of Pseudomonas saccharophila. J. biol. Chem. 196, 853.

Forkner, C. E. (1960). Pseudomonas aeruginosa Infections. New York: Grune and Stratton.

Forsyth, W. G. C., Hayward, A. C. \& Roberts, J. B. (1958). Occurrence of poly- $\beta$ hydroxybutyric acid in aerobic Gram-negative bacteria. Nature, Lond. 182, 800.

Gutner, L. B. \& Fisher, M. W. (1948). Chronic melioidosis : discussion, case and report, and special studies. Ann. Intern. Med. 28, 1157. 
Haupt, H. (1957). In Bergey's Manual of Determinative Bacteriology, 7th ed. p. 417. Baltimore: Williams and Wilkins.

Haynes, W. C. (1957). In Bergey's Manual of Determinative Bacteriology, 7th ed. p. 100. Baltimore: Williams and Wilkins.

Houden, M. (1935). Loefflerella: glanders and melioidosis. In Gay, F. P. et al. Agents of Disease and Host Resistance. Springfield, : C. C. Thomas.

JESSEN, O. (1965). Pseudomonas aeruginosa and other green fluorescent pseudomonads. $A$ taxonomic study. Copenhagen: Munksgaard.

Konovaltschikoff-Mazoyer, M. \& Senez, J. C. (1956). Dégradation bactérienne des hydrocarbures paraffiniques. I. Isolement et caractérisation de souches marines et terrestres appartenant au genre Pseudomonas. Ann. Inst. Pasteur. 91, 60.

Lajudie, P., Fournier, J. \& Chambon, L. (1953). L'appareil flagellaire du bacille de Whitmore. Ann. Inst. Pasteur. 85, 112.

Leifson, E. (1960). Atlas of Bacterial Flagellation. New York: Academic Press.

Levine, H. B., Dowling, J. H., Evenson, M. \& Lien, O. G. (1954). Growth of Malleomyces pseudomallei in simple chemically defined media. $J$. Bact. 67, 350 .

Levine, H. B. \& Wolochow, H. (1960). Occurrence of poly- $\beta$-hydroxybutyrate in Pseudomonas pseudomallei. J. Bact. 79, 305.

Loeffler \& Schütz (1882). Dt. med. Wschr. 8, 707. Cited by Holden (1935).

LwoFf, A. (1944). L'Evolution physiologique. Paris: Hermann.

Lysenko, O. (1961). Pseudomonas - an attempt at a general classification. J. gen. Microbiol. $25,379$.

Mandel, M. (1966). Deoxyribonucleic acid base composition in the genus Pseudomonas. J. gen. Microbiol. 43, 273.

Mirler, W. R., Pannell, L., Cravitz, L., Tanner, W. A. \& Rosebury, T. (1948). Studies on certain biological characteristics of Malleomyces mallei and Malleomyces pseudomallei. II. Virulence and infectivity for animals. $J$. Bact. 55, 127.

NigG, C. (1963). Serologic studies on subclinical melioidosis. J. Bact. 91. 18.

Nrgg, C. \& Johnston, M. M. (1961). Complement fixation test in experimental clinical and subclinical melioidosis. J. Bact. 82, 159.

Nigg, C., Ruch, J., Scott, E. \& Noble, K. (1956). Enhancement of virulence of Malleomyces pseudomallei. J. Bact. 71, 530.

Pribram, E. (1933). Klassifikation der Schizomyceten. Leipzig und Wien: F. Deuticke.

Rimington, R. A. (1962). Melioidosis in North Queensland. Med. J. Aust. 49, 50.

Roques \& Dauphin (1943). Mélioidose post-operatoire et localization chirurgicale. Rev. méd. fr. Extr.-Orient, 21, 267. Cited by Nigg \& Johnston (1961).

Smith, P. B. \& Cherry, W. B. (1957). Identification of Malleomyces by specific bacteriophages. $J$. Bact. 74, 668 .

Stanier, R. Y., Palleroni, N. J. \& Doudonoff, M. (1966). The aerobic pseudomonads: A taxonomic study. J. gen. Microbiol. 43, 159.

Stanton, A. T. \& Fletcher W. (1925). Melioidosis and its relation to glanders. J. Hyg., Camb. 347.

Steel, K. J. \& Cowan, S. T. (1964). Le rattachement de Bacterium anitratum, Moraxella lwoffi, Bacillus mallei et Haemophilus parapertussis au genre Acinetobacter Brisou et Prévot. Ann. Inst. Pasteur, 106, 479.

Sutmöller, P., Kraneveld, F. C. \& Schaaf, A. v.d. (1957). Melioidosis (Pseudomalleus) in sheep, goats and pigs on Aruba (Netherlands Antilles). J. Am. vet. med. Ass. 130, 415.

Topley and Wilson's Principles of Bacteriology and Immunity, (1955). 4th ed. Ed. by G. S. Wilson and A. A. Miles, Baltimore: Williams and Wilkins.

Wetmore, P. W. \& Gochenour, W. S. (1956). Comparative studies of the genus Malleomyces and selected Pseudomonas species. J. Bact. 72, 79.

Whitmore, A. (1913). An account of a glanders-like disease occurring in Rangoon. J. Hyg., Camb. 13, 1.

Ziskind, J., Pizzolato, P. \& Buff, E. E. (1954). Characteristics of a strain of Malleomyces pseudomallei from chronic melioidosis. Am. J. clin. Path. 24, 1241.

Zopf, W. F. (1885). Die Spaltpilze. Breslau: E. Trewendt. 
Note added to proofs

Antisera from rabbits hyperimmunized with formalin-killed cells of both Pseudomonas pseudomallei NBL 114 and Bacillus mallei NBL 7 were used to perform slideagglutination tests with all strains of both species. Each antiserum agglutinated to at least the 1/50 dilution all strains of both $P$. pseudomallei and B. mallei. Serum from a rabbit hyperimmunized with formalin-killed cells of $\boldsymbol{P}$ seudomonas aeruginosa NCTC 9229 did not agglutinate any strain of $P$. pseudomallei and $B$. mallei at the 1/10 dilution and neither did antisera to $P$. pseudomallei NBL 114 and $B$. mallei NBI 7 agglutinate $P$. aeruginosa NCTC 9229 at the same dilution. No strain of any of the three species was agglutinated by normal rabbit antisera diluted 1/10. 